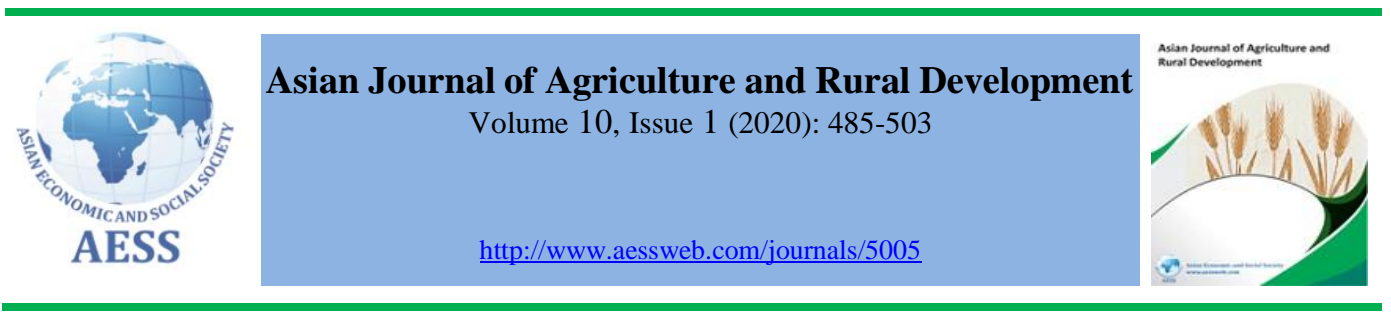

\title{
GGE BIPLOT ANALYSIS OF MULTI-LOCATION YIELD TRIAL OF GINGER (Zingiber officinale Rosc.) GENOTYPES IN SOUTH-EASTERN, NIGERIA
}

Abua, Mary Njei ${ }^{\mathrm{a}}$,
Iwo, Godfery Akpan',
Ittah, Macauley Asim
Obok, Ekemini Edet
Edugbo, Richmond Emu

ARTICLE HISTORY:

Received: 11-Mar-2020

Accepted: 18-May-2020

Online Available: 29-Jun-

2020

\section{Keywords:}

Genotype

Genotype by environment,

Multi-location trial,

Mega-environment,

GGE biplot analysis and

yield performance
${ }^{\text {a }}$ Department of Crop Science, University of Calabar, Calabar, Cross River State, Nigeria

${ }^{\mathrm{b}}$ Department of Agronomy, Micheal Okpara University of

Agriculture, Umudike, Abia State, Nigeria

- marynjei@yahoo.com;njeiabua@unical.edu.ng;

maryabua44@gmail.com (Corresponding author)

\begin{abstract}
Multi-location trials were conducted in 2016 and 2017 at Calabar, Ikom and Ogoja in Cross River State, Nigeria, to determine the yield stability of 17 ginger genotypes (G1-G17) using genotype and genotype by environment (GGE) biplot model. The location and year combination gave six environments (E1-E6). The experiment was laid out in split plots using a randomized complete block design with three replications. Yield related traits like rhizome fingers, rhizome length, and rhizome yield were determined. E3 (i.e. Ikom in 2016) was ranked as the ideal environment for ginger production in Cross River State. While G5 (UGI-5-04) was classified as the ideal genotype for rhizome yield in Calabar, Ikom and Ogoja. Ikom in 2016 (E3) and 2017 (E4) were identified as megaenvironments for UG1-13-02, UG1-5-04, UG1-5-18, UG1-5-35, UG1-538, UG2-11-03 and UG2-9-01 while Ogoja in 2016 (E5) and 2017 (E6) were identified as mega-environments for UG1-2-35, UG1-5-48, UG1-552 and UG1-7-24 ginger mutants. Ikom is recommended as a suitable environment for the cultivation of generally adapted ginger genotypes namely, UG1-5-04, UG1-5-38, UG1-13-02 and UG2-9-01). Ogoja was suitable for specific adaptation of UG1-7-24 and UG1-5-48 ginger mutants. These mutants are recommended for consideration in subsequent ginger breeding and improvement programmes.
\end{abstract}

\section{Contribution/ Originality}

The research explored that the Ikom region has an ideal environment for the cultivation of ginger in Cross River State. Different varieties of ginger mutants were identified as ideal genotypes in other regions like Ogoja and Calabar.

DOI: 10.18488/journal.1005/2020.10.1/1005.1.485.503

ISSN (P): 2304-1455/ISSN (E):2224-4433

How to cite: Abua, Mary Njei, Iwo, Godfery Akpan, Ittah, Macauley Asim, Obok, Ekemini Edet and Edugbo, Richmond Emu (2020). GGE biplot analysis of multi-location yield trial of ginger (Zingiber Officinale Rosc.) genotypes in South-Eastern, Nigeria. Asian Journal of Agriculture and Rural Development, 10(1), 485-503.

(c) 2020 Asian Economic and Social Society. All rights reserved. 


\section{INTRODUCTION}

Ginger (Zingiber officinale Rosc.) is a monocotyledonous perennial herb in the family Zingiberaceae, grown mainly for its spicy and aromatic rhizomes (Peter et al., 2007). It is a valued export crop in Nigeria whose demand in the international market is high because of its rich nutrient profile, essential oils, and oleoresin contents (Adegboye, 2011; Abua et al., 2020). Globally, the yearly demand for ginger is increasing due to its diverse utilization (FAO, 2017). The high demand for ginger in the international market makes it an important export crop for Nigeria, and thus serves as a source of foreign exchange earnings. In Nigeria, the demand for this crop is on a steady increase and the solution to this challenge is to increase its production. This requires the cultivation of improved ginger varieties with enhanced economic yields.

Ginger has a narrow genetic base because it is vegetatively propagated (Babu et al., 2013). Conventional breeding procedures like hybridization is difficult for ginger improvement. Therefore, an alternative means for improving the crop is to create variability through induced mutation. The induced mutation has been considered an established method for increasing genetic variability in many crops. FAO (2006), documented 2,540 varieties of crops that have been commercially released through mutation breeding, for example, garlic, cassava, turmeric, and potato.

In this study, fifteen ginger lines were developed through mutation breeding (gamma-ray exposure) using two ginger landraces (UG1 and UG2). Therefore, there is a need for these new ginger mutant lines to be evaluated across several locations to determine their yield potentials, superiority as well as their yield stability before their release for wide-range cultivation. This has prompted the establishment of this research in three agro-ecological locations across Cross River State, Nigeria.

Multi-environment trials (METs) are mostly carried out by plant breeders to evaluate improved genotypes across test environments (several locations and over years) before a specific genotype is recommended to growers for commercial production. Most genotypes show crossover interactions (i.e. do not have uniform performance in all environments); by implication, one genotype may have the highest yield in some environments and the second genotype may be excellent in other environments (Zahia et al., 2010). The development of genotypes that can be adapted to a wide range of different environments is the ultimate goal of plant breeders in crop improvement programmes (Ahmadi et al., 2015). METs are conducted to identify locations that best represent the target environments and to identify the best genotypes or cultivars suited for recommendations to farmers (Odor et al., 2017; Vaezi et al., 2017). Multi-Environment Trials (METs) are important for studying yield stability, adaptation and for a prediction of yield performance of crop genotypes across environments (Yan and Kang, 2003; Dehghani et al., 2009). It is difficult to identify superior genotypes due to the genotypes $\times$ environments $(G \times E)$ interaction, but assessing such interaction is extremely important since it is the number one factor responsible for changing genotype performance in different environments (Mohamed, 2013, Oliveira et al., 2017). G $\times \mathrm{E}$ interaction studies allow for the identification of the ideal location(s) to each genotype which would maximize yield potential and reduce production costs (Oyekunle et al., 2017). Information on the structure and nature of $\mathrm{G} \times \mathrm{E}$ interaction is particularly useful to plant breeders because it can help determine if they need to develop cultivars for all environments of interest or if they should develop specific cultivars for specific target environments.

Data from multi-environment trials (METs) are usually quite large. It is difficult to understand the pattern of the data without some kind of graphical presentation. The biplot technique by Gabriel (1971) provides a solution to this challenge. A biplot that displays the GGE of a MET data, referred to as a GGE biplot (graphical method), is an ideal tool for MET data analysis (Yan et al., 2000; Yan, 2001). The GGE biplot analysis is efficient because it allows predictions to be made on the mean genotype yield per specific environment, as well as helps identify the most stable genotype for the region of interest (Yan, 2014, Santos et al., 2017). Genotype and genotype by environment 
(GGE) biplot analysis consists of a set of graphs that allow visualization of the patterns in a dataset from different angles (Yan and Kang, 2003; Yan and Tinker, 2006; Yan, 2014).

A GGE biplot as a data visualization tool can graphically demonstrate a $(\mathrm{G} \times \mathrm{E})$ interaction pattern, it is an effective tool used for: (i) mega environment analysis (e.g. "Which-Won-Where" pattern), whereby specific genotypes can be recommended to specific mega-environments (Yan, 2014), (ii) genotype evaluation (the mean performance and stability), and (iii) environmental evaluation (the power to discriminate among genotypes in target environments. The objectives of this study were (i) to display graphically, the performance and stability of 17 ginger lines across three locations in two years, (ii) to identify ginger lines with wide or specific adaptation and (iii) to identify the ideal environment for ginger cultivation among the three test locations.

\section{MATERIALS AND METHODS}

This experiment was carried out at Calabar, Ikom, and Ogoja in Cross River State, Nigeria in 2016 and 2017 cropping seasons. These locations were chosen based on their unique differences in edaphic and climatic conditions such as physicochemical properties of the soil, rainfall, relative humidity, temperature, and sunshine duration.

Seventeen ginger genotypes consisting of fifteen (15) mutant lines (UG1-11-07, UG1-13-02, UG12-35, UG1-5-04, UG1,-5-18, UG1-5-22, UG1-5-31, UG1-5-35, UG1-5-38, UG1-5-48, UG1-5-49, UG1-5-52, UG1-7-24, UG2-9-01 and UG2-11-03) and two local check landraces (UG1 and UG2) were sourced from National Root Crop Research Institute (NRCRI), Umudike, Nigeria. The fifteen mutant lines were derived from the existing local varieties UG1 and UG2 by exposing them to different doses of gamma-ray irradiation. The mutant lines derived from UG1 were exposed to $2 \mathrm{GY}, 5 \mathrm{GY}, 7 \mathrm{GY}, 11 \mathrm{GY}$, and $13 \mathrm{GY}$ doses of gamma-ray to give the following mutant lines: UG12-35, UG1-5-04, UG1-5-18, UG1-5-22, UG1-5-31, UG1-5-35, UG1-5-38, UG1-5-48, UG1-5-49, UG1-5-52, UG1-7-24, UG1-11-07 and UG1-13-02. The mutant lines derived from UG2 were exposed to $9 \mathrm{GY}$ and $11 \mathrm{GY}$ doses of gamma-ray to give the following mutant lines: UG1-9-01 and UG2-11-03.

This experiment was a split-plot laid out in a randomized complete block design (RCBD) with three replications. Location served as whole plot treatment while the ginger lines served as the sub-plot. The following yield traits were measured: number of rhizome fingers per plant, rhizome length $(\mathrm{cm})$, and rhizome yield (t/ha). Stability analysis was carried out using Genotype and Genotype by Environment (GGE) biplot model according to Yan (2001) and the model was depicted as follows:

$$
\mathrm{Y}_{i j}=\mu+\alpha_{i}+\beta_{j}+\mathrm{j}_{i j}
$$

Where $Y_{i j}$ is the measure of the $i$ th genotype in the $j$ th environment; $\mu$ is the grand mean, $\alpha_{i}$ is the main effect of the $i$ th genotype; $\beta_{j}$ is the main effect of the $j$ th environment, $\mathrm{j}_{i j}$ is the interaction between the $i$ th genotype and $j$ th environment. The 17 ginger lines served as the genotype while the three locations and the two years formed a combination of six (6) environments.

\section{RESULTS AND DISCUSSION}

\subsection{GGE biplot analysis for the number of rhizome fingers per plant}

The genotype and genotype by environment (GGE) biplot captured $79.7 \%$ of the genotype main effect and the genotype by environment interaction (GE) interaction with the principal component 1 (PC1) and principal component 2 (PC2) axes explaining $54.4 \%$ and $25.3 \%$ respectively. Figure 1 displays the GGE biplot environment vector view for the number of rhizome fingers per plant. The biplot shows the relationship among the test environments as well as their discriminating ability. The angle between the vectors of any two environments estimates the correlation between them. 
The larger the angle the less correlated the environments (Yan and Tinker, 2006) The biplot indicated that Ikom in 2016 (E3) and Ikom in 2017 (E4) were closely correlated. Ogoja in 2016 (E5) and Ogoja in 2017 (E6) also had strong correlations. The discriminating ability of the test environments is estimated by the length of their vectors. (Yan, 2001). Ogoja in 2017 (E6) (E6) was selected as the most discriminating environment followed by Ogoja in 2016 (E5) while Calabar in 2016 (E2) was the least discriminating environment for this trait.

Table 1: Physicochemical properties of the soil and weather conditions at the test locations

\begin{tabular}{lccccccccc}
\hline Year/Location & $\begin{array}{c}\text { Sand } \\
(\boldsymbol{\%})\end{array}$ & $\begin{array}{c}\text { Silt } \\
(\boldsymbol{\%})\end{array}$ & $\begin{array}{c}\text { Clay } \\
(\boldsymbol{\%})\end{array}$ & $\begin{array}{c}\mathbf{p H} \text { in } \\
\mathbf{H}_{\mathbf{2}} \mathbf{O}\end{array}$ & $\mathbf{E C E C}$ & $\begin{array}{c}\text { Temp. } \\
\left({ }^{\mathbf{}} \mathbf{C}\right)\end{array}$ & $\begin{array}{c}\text { Relative } \\
\text { Humidity } \\
(\boldsymbol{\%})\end{array}$ & $\begin{array}{c}\text { Rainfall } \\
(\mathbf{m m})\end{array}$ & $\begin{array}{c}\text { Sunshine } \\
(\mathbf{h})\end{array}$ \\
\hline 2016 & & & & \multicolumn{7}{c}{ Mean } \\
Calabar & - & - & - & - & - & 31.7 & 85.6 & 190.7 & 3.43 \\
Ikom & - & - & - & - & - & 30.5 & 83.1 & 210.4 & 5.62 \\
Ogoja & - & - & - & - & - & 33.5 & 71.3 & 146.6 & 5.91 \\
2017 & & & & & & Mean & & & \\
Calabar & 79.0 & 13.0 & 8.0 & 5.7 & 7.94 & 31.3 & 86.8 & 260 & 3.58 \\
Ikom & 47.0 & 22.0 & 31.0 & 5.8 & 11.74 & 32.5 & 82.4 & 189.5 & 5.15 \\
Ogoja & 73.0 & 17.0 & 10.0 & 5.2 & 5.57 & 34.4 & 71.3 & 146.6 & 4.94 \\
\hline
\end{tabular}

ECEC: Effective Cation Exchange Capacity

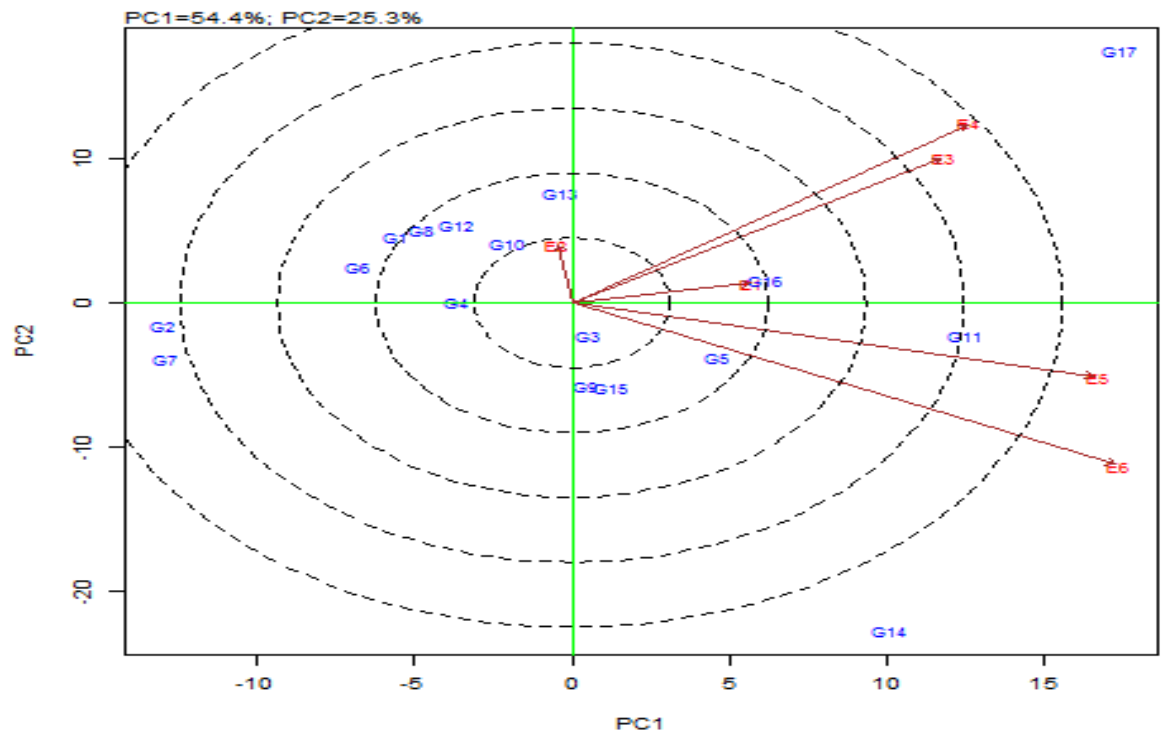

Figure 1: Relationship among the test environments ands their discriminating ability for the number of rhizome fingers per plant

Key: G1 = UG1; G2 = UG1-11-07; G3 = UG1-13-02; G4 = UG1-2-35; G5 = UG1-5-04; G6 = UG1-5-18; G7 = UG1-5-22; G8 = UG1-5-31; G9 = UG1-5-35; G10 = UG1-5-38; G11 = UG1-5-48; G12 = UG1-5-49; G13 = UG1-5-52; G14 = UG1-7-24; G15 = UG2; G16 = UG2-11-03; G17 = UG2-9-01; E1 = Calabar 2016; E2 = Calabar 2017; E3 = Ikom 2016; E4 = Ikom 2017; E5 = Ogoja 2016; E6 = Ogoja 2017

The GGE biplot environment view showing the representativeness of the test environments as well as the ranking of the environments to the 'ideal test environment' is presented in Figure 2. The representativeness evaluation of the test environments is the second most important aspect of test 
environments after the discriminating ability evaluation. The representativeness of the test environment is estimated by the size of the angle of its vector to the AEA (Average Environment Axis). The smaller the angle of the environment vectors to the AEA the more representative the environment would be of the test environments (Yan, 2001). Thus, Calabar in 2016 (E1) is the most representative of the test environments while Calabar in 2016 (E2) is the least representative environment. The ideal test environment should be the most discriminating (informative) and also the most representative of the target environment. The "ideal test environment" is indicated by the centre of the concentric circles. It is a point on the AEA in the positive direction ("most representative") with a distance to the biplot origin equal to the longest vector of all environments ("most informative"). Ogoja in 2016 (E5) is the closest to this point while Calabar in 2016 (E2) was the furthest from this point.

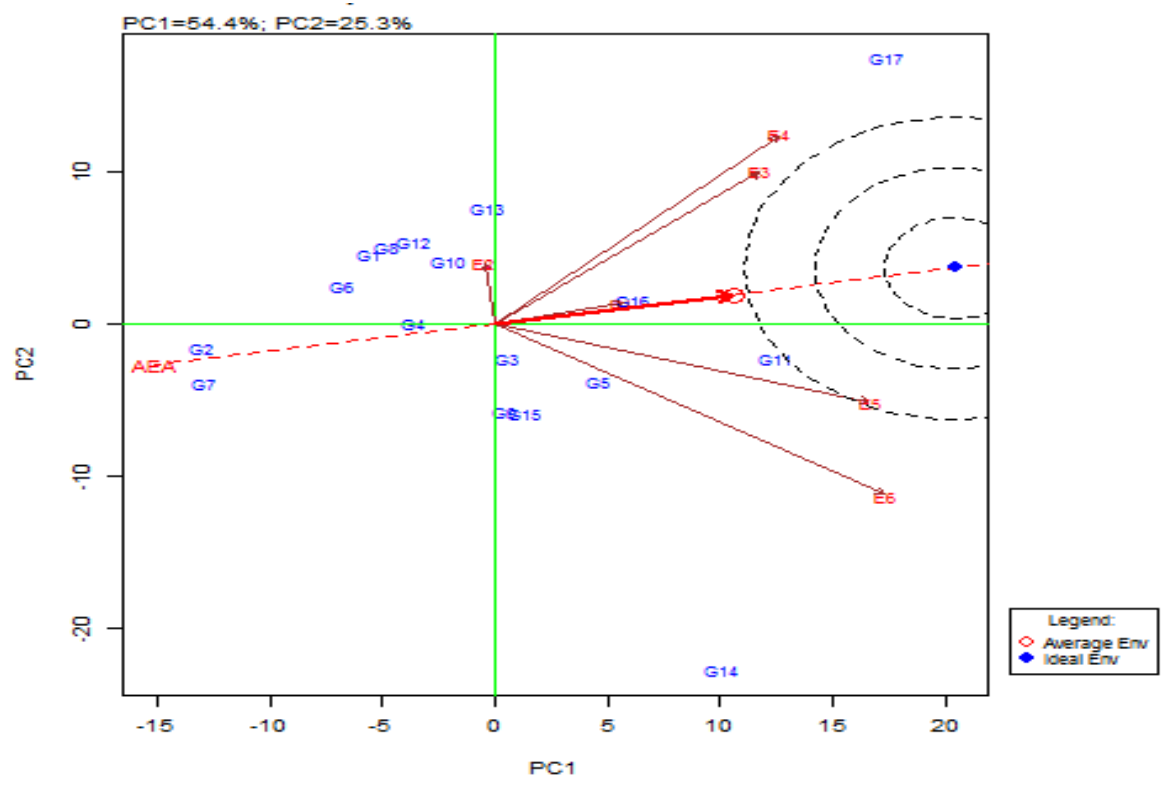

Figure 2: Representativeness and ranking of the environments for a number of rhizome fingers per plant

Key: G1 = UG1; G2 = UG1-11-07; G3 = UG1-13-02; G4 = UG1-2-35; G5 = UG1-5-04; G6 = UG1-5-18; G7 = UG1-5-22; G8 = UG1-5-31; G9 = UG1-5-35; G10 = UG1-5-38; G11 = UG1-5-48; G12 = UG1-5-49; G13 = UG1-5-52; G14 = UG1-7-24; G15 = UG2; G16 = UG2-11-03; G17 = UG2-9-01; E1 = Calabar 2016; E2 = Calabar 2017; E3 = Ikom 2016; E4 = Ikom 2017; E5 = Ogoja 2016; E6 = Ogoja 2017

Figure 3 shows the GGE biplot genotype view indicating the relationship among the seventeen ginger lines (genotypes). Genotypes are positively correlated with one another if the angle between each of their vectors is less than $90^{\circ}$. The biplot showed that G17 (UG2-9-01) and G16 (UG2-1103) were closely correlated while G3 (UG1-13-02), G9 (UG1-5-35), G15 (UG2) and G14 (UG1-724) were closely correlated. Close correlations were also seen between G5 (UG1-5-04) and G11 (UG1-5-48). Close correlations also existed between G1 (UG1), G2 (UG1-11-07), G4 (UG1-2-35), G6 (UG1-5-18), G7 (UG1-5-22), G8 (UG1-5-31), G10 (UG1-5-38) and G12 (UG1-5-49). 


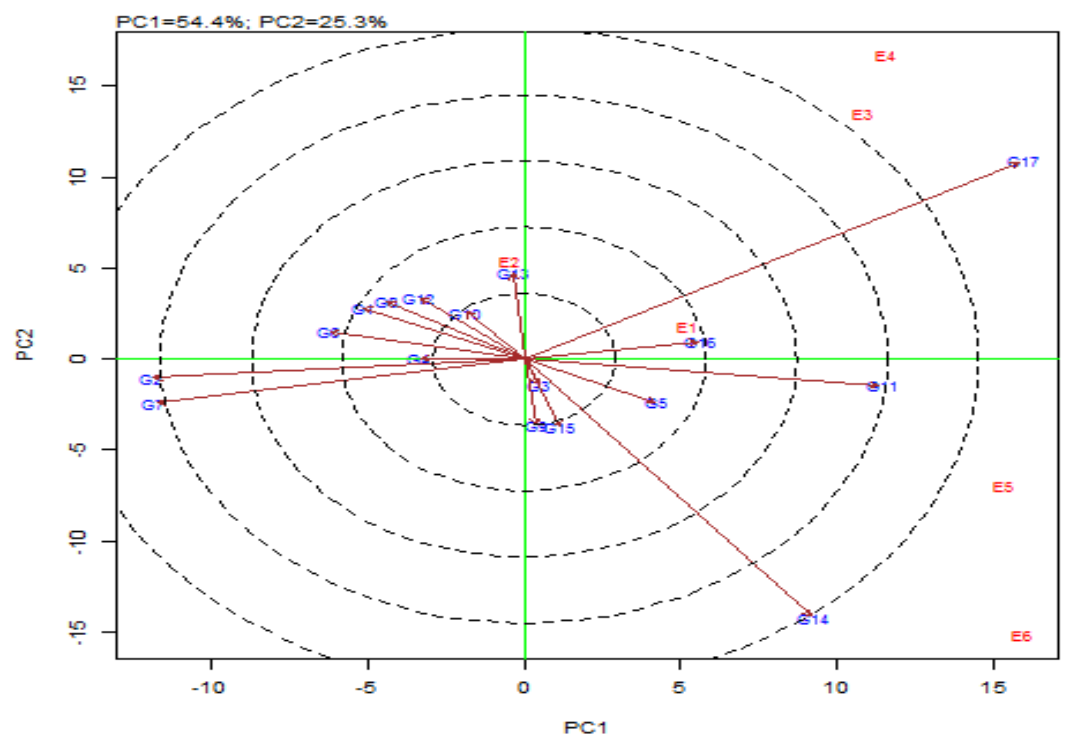

Figure 3: Relationship among the genotype for the number of rhizome fingers per plant

Key: G1 = UG1; G2 = UG1-11-07; G3 = UG1-13-02; G4 = UG1-2-35; G5 = UG1-5-04; G6 = UG1-5-18; G7 = UG1-5-22; G8 = UG1-5-31; G9 = UG1-5-35; G10 = UG1-5-38; G11 = UG1-5-48; G12 = UG1-5-49; G13 = UG1-5-52; G14 = UG1-7-24; G15 = UG2; G16 = UG2-11-03; G17 = UG2-9-01; E1 = Calabar 2016; E2 = Calabar 2017; E3 = Ikom 2016; E4 = Ikom 2017; E5 = Ogoja 2016; E6 = Ogoja 2017

Figure 4 shows the ranking of the genotypes for both mean performance and stability for the number of rhizome fingers per plant across the six environments. G17 (UG2-9-01) was selected as the best performing genotype and relatively stable for the trait and thus ranked closest to the ideal genotype. This was followed by G11 (UG1-5-48). G16 (UG2-11-03) was highly stable for the trait but its mean performance was lower than that of G17 (UG2-9-01) and G11 (UG1-5-48). G2 (UG111-07) and G7 (UG1-5-22) were the poorest performers and were ranked furthest away from the ideal genotype although they exhibited high stability for the character.

Figure 5 displays the "what-won-where" GGE biplot for the number of rhizome fingers per plant. Three projecting lines divided the biplot into three sectors. The most responsive genotypes are placed on the vertex of the polygon. In other words, genotypes located on the vertices of the polygon performed either the best or the poorest in one or more environments. The biplot selected G17 (UG2-9-01) as the winner in four of the six environments while G14 (UG1-7-24) was the winner in Ogoja in 2016 (E5) and Ogoja in 2017 (E6) (E6). G2 (UG1-11-03) and G7 (UG1-5-22) were selected as the poorest performers across all six environments. 


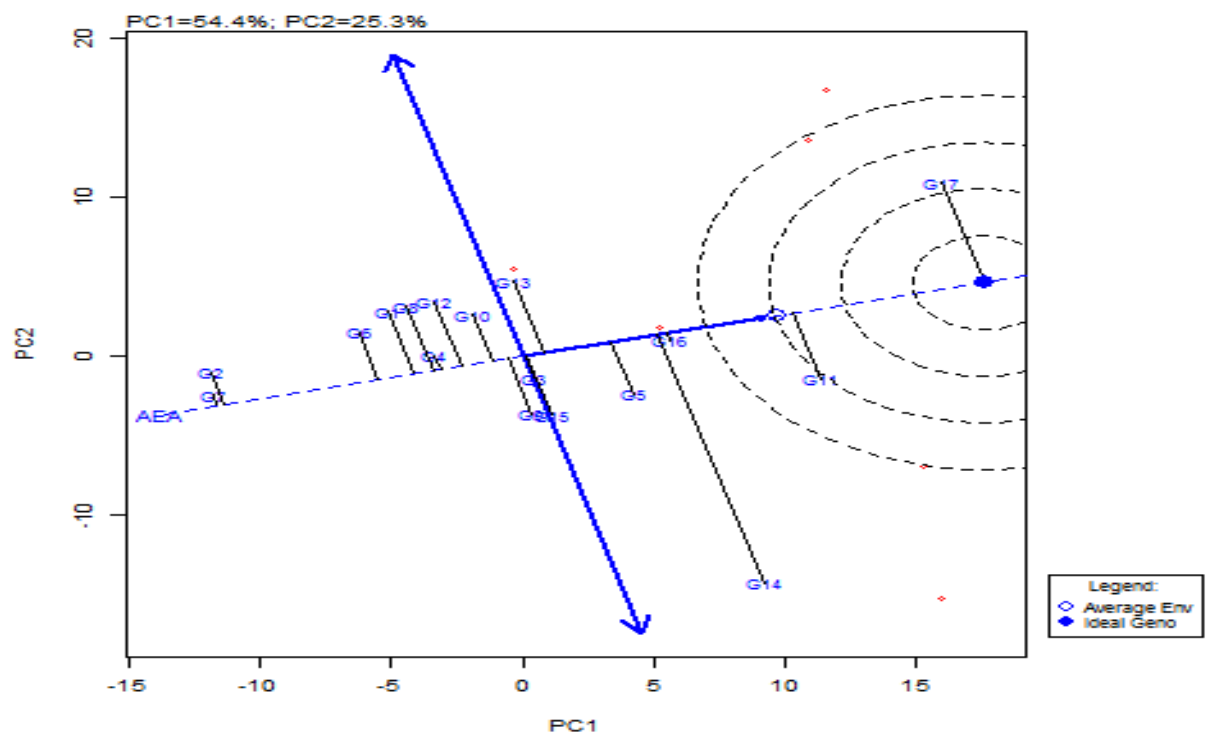

Figure 4: Mean performance and stability for the number of rhizome fingers per plant

Key: G1 = UG1; G2 = UG1-11-07; G3 = UG1-13-02; G4 = UG1-2-35; G5 = UG1-5-04; G6 = UG1-5-18; G7 = UG1-5-22; G8 = UG1-5-31; G9 = UG1-5-35; G10 = UG1-5-38; G11 = UG1-5-48; G12 = UG1-5-49; G13 = UG1-5-52; G14 = UG1-7-24; G15 = UG2; G16 = UG2-11-03; G17 = UG2-9-01. E1 = Calabar 2016; E2 = Calabar 2017; E3 = Ikom 2016; E4 = Ikom 2017; E5 = Ogoja 2016; E6 = Ogoja 2017

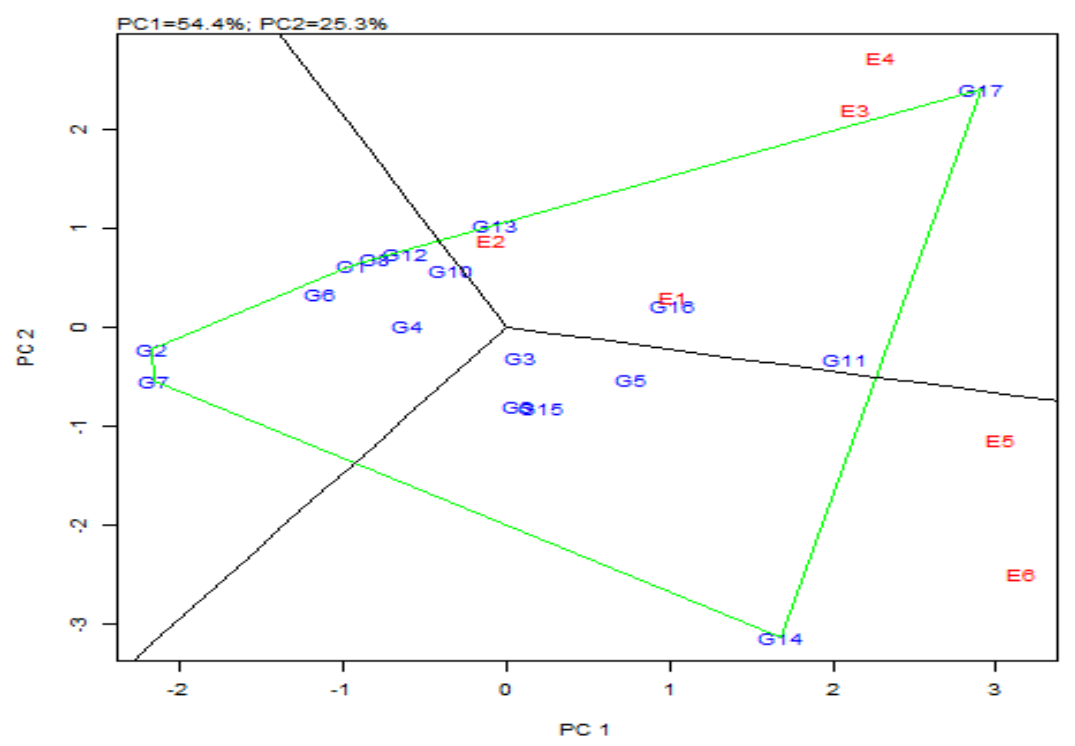

Figure 5: "What-Won-Where" GGE biplot for number of rhizome finger per plant

Key: G1 = UG1; G2 = UG1-11-07; G3 = UG1-13-02; G4 = UG1-2-35; G5 = UG1-5-04; G6 = UG1-5-18; G7 = UG1-5-22; G8 = UG1-5-31; G9 = UG1-5-35; G10 = UG1-5-38; G11 = UG1-5-48; G12 = UG1-5-49; G13 = UG1-5-52; G14 = UG1-7-24; G15 = UG2; G16 = UG2-11-03; G17 = UG2-9-01. E1 = Calabar 2016; E2 = Calabar 2017; E3 = Ikom 2016; E4 = Ikom 2017; E5 = Ogoja 2016; E6 = Ogoja 2017 


\subsection{GGE biplot analysis for rhizome length $(\mathrm{cm})$}

GGE biplot for rhizome length captured $83.7 \%$ of variations due to the genotype main effect and G $\times$ E interaction. PC1 explained 70.4\% while PC2 explained 13.3\%. Figure 6 displays the GGE biplot environment vector view for rhizome length. The biplot showed that the environments fell into three groups. Ogoja in 2016 (E5) and Ogoja in 2017 (E6) were closely related and had similar interaction patterns. Ikom in 2016 (E3) Ikom in 2017 (E4) was closely correlated and had a similar interaction pattern. Calabar in 2016 (E1) and E2 Calabar in 2017 (E2) were not correlated. The biplot also showed Ogoja in 2016 (E5) as the most discriminating test environment as it had the longest vector from the origin of the biplot while Calabar in 2016 (E1) was the least discriminating environment.

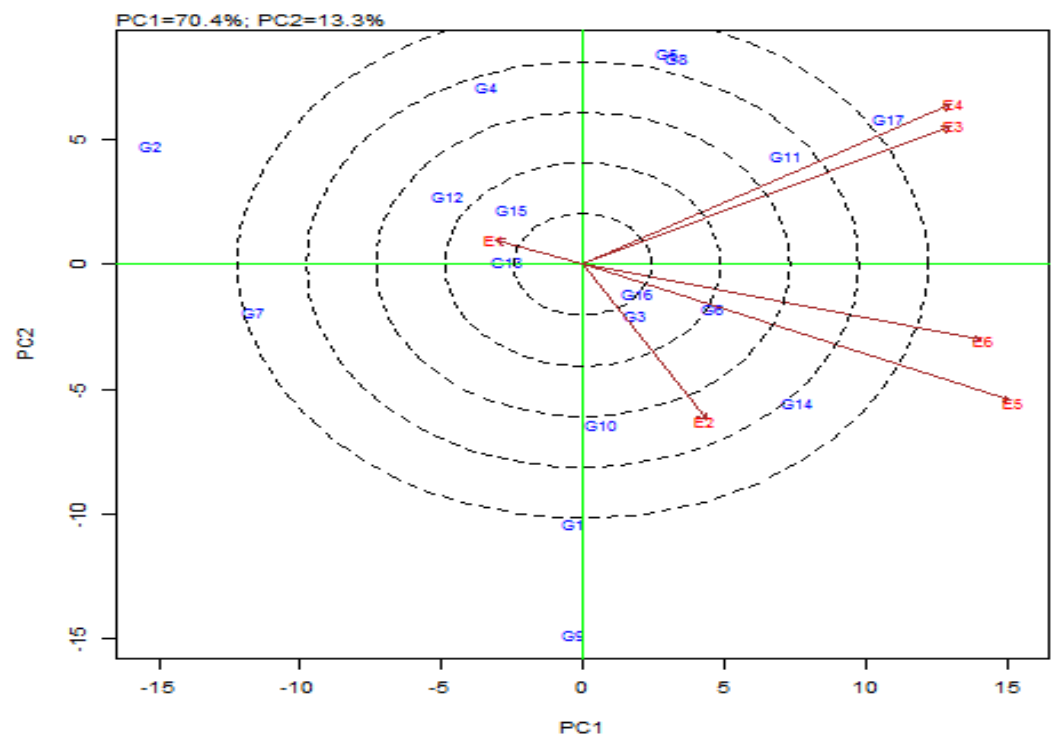

Figure 6: Relationship among the test environments and their discriminating ability for rhizome length $(\mathrm{cm})$

Key: G1 = UG1; G2 = UG1-11-07; G3 = UG1-13-02; G4 = UG1-2-35; G5 = UG1-5-04; G6 = UG1-5-18; G7 = UG1-5-22; G8 = UG1-5-31; G9 = UG1-5-35; G10 = UG1-5-38; G11 = UG1-5-48; G12 = UG1-5-49; G13 = UG1-5-52; G14 = UG1-7-24; G15 = UG2; G16 = UG2-11-03; G17 = UG2-9-01. E1 = Calabar 2016; E2 = Calabar 2017; E3 = Ikom 2016; E4 = Ikom 2017; E5 = Ogoja 2016; E6 = Ogoja 2017

Figure 7 displays the GGE ranking biplot for the environment as well as the evaluation of their representativeness. Ogoja in 2017 (E6) was the most representative of the test environments as its vector had the smallest angle to the AEA. The biplot also ranked Ogoja in 2017 (E6) as the preferred test environment as it was closest to the ideal environment followed by Ogoja in 2016 (E5). Calabar in 2016 (E1) was ranked last.

Figure 8 displays the GGE biplot genotype view for rhizome length. The biplot shows the relationship among the genotypes. Genotypes with vectors at close angles have similar responses across the environments. Close correlations were shown between G17 (UG2-9-01) and G11 (UG15-48); G6 (UG1-5-48) and G14 (UG1-7-24); G1 (UG1) and G9 (UG1-5-35) and between G2 (UG1-11-07) and G7 (UG1-5-22). 


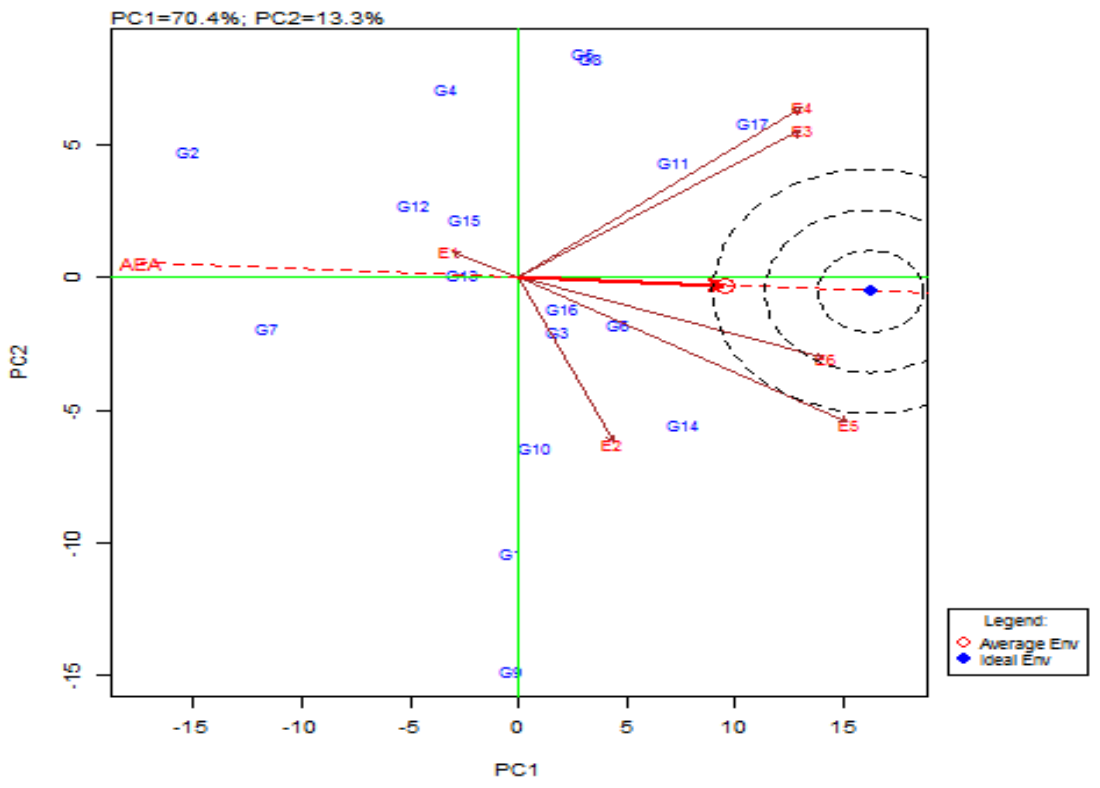

Figure 7: Representativeness and ranking of the environments for rhizome length $(\mathrm{cm})$

Key: G1 = UG1; G2 = UG1-11-07; G3 = UG1-13-02; G4 = UG1-2-35; G5 = UG1-5-04; G6 = UG1-5-18; G7 = UG1-5-22; G8 = UG1-5-31; G9 = UG1-5-35; G10 = UG1-5-38; G11 = UG1-5-48; G12 = UG1-5-49; G13 = UG1-5-52; G14 = UG1-7-24; G15 = UG2; G16 = UG2-11-03; G17 = UG2-9-01; E1 = Calabar 2016; E2 = Calabar 2017; E3 = Ikom 2016; E4 = Ikom 2017; E5 = Ogoja 2016; E6 = Ogoja 2017

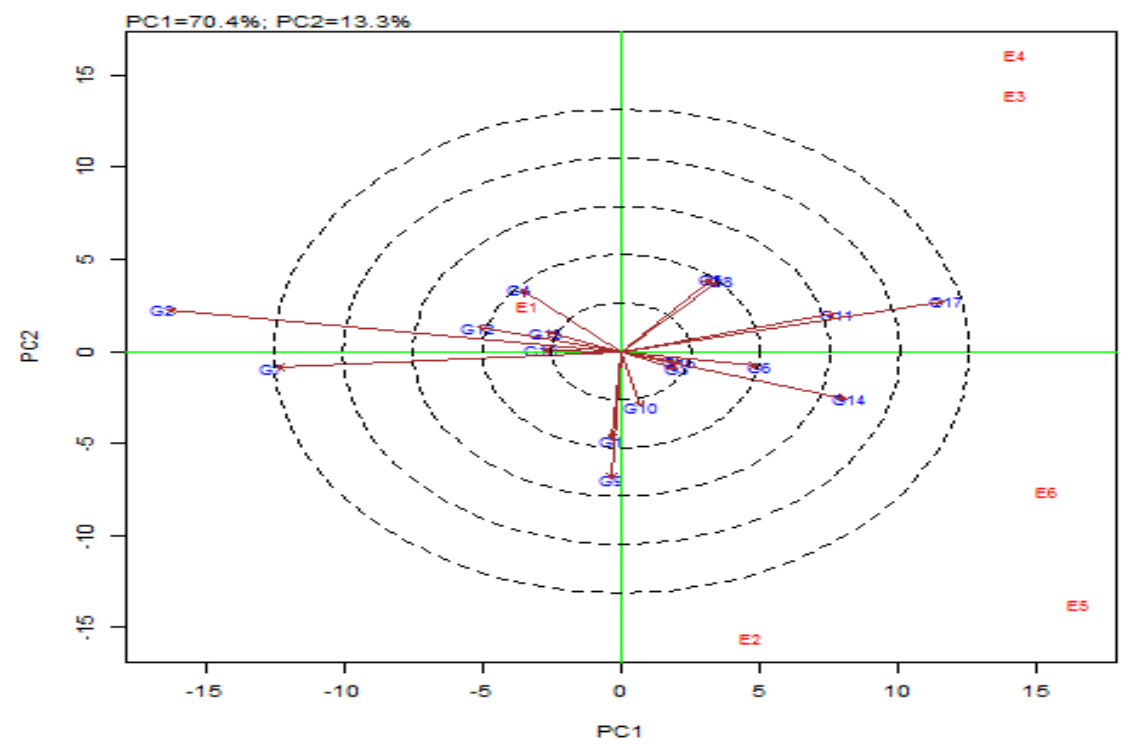

Figure 8: Relationship among the genotype for rhizome length $(\mathrm{cm})$

Key: G1 = UG1; G2 = UG1-11-07; G3 = UG1-13-02; G4 = UG1-2-35; G5 = UG1-5-04; G6 = UG1-5-18; G7 = UG1-5-22; G8 = UG1-5-31; G9 = UG1-5-35; G10 = UG1-5-38; G11 = UG1-5-48; G12 = UG1-5-49; G13 = UG1-5-52; G14 = UG1-7-24; G15 = UG2; G16 = UG2-11-03; G17 = UG2-9-01; E1 = Calabar 2016; E2 = Calabar 2017; E3 = Ikom 2016; E4 = Ikom 2017; E5 = Ogoja 2016; E6 = Ogoja 2017 
Figure 9 displays the GGE biplot genotype ranking view for both mean performance and stability for rhizome length (cm). G1 (UG2-9-01), G14 (UG1-7-24), and G11 (UG1-5-48) were the best in terms of mean performance. However, G13 (UG1-5-52), G6 (UG1-5-18), and G16 (UG2-11-03) were the most stable for the trait. In the ranking of the genotypes, G17 (UG2-9-01) was ranked closest to the "ideal genotype" followed by G14 (UG1-7-24) and G11 (UG1-5-38). G2 (UG1-1107 ) and G7 (UG1-5-22) had the poorest mean performance and were the least ranked genotypes.

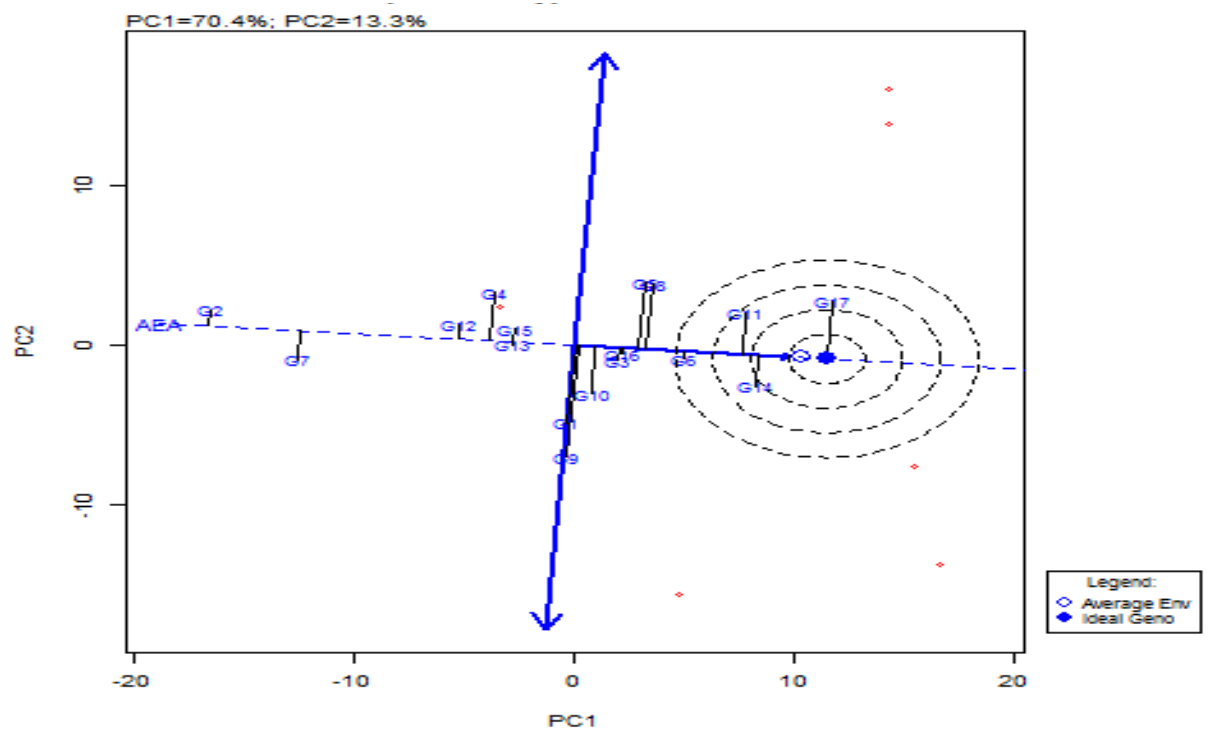

Figure 9: Mean performance and stability for rhizome length $(\mathrm{cm})$

Key: G1 = UG1; G2 = UG1-11-07; G3 = UG1-13-02; G4 = UG1-2-35; G5 = UG1-5-04; G6 = UG1-5-18; G7 = UG1-5-22; G8 = UG1-5-31; G9 = UG1-5-35; G10 = UG1-5-38; G11 = UG1-5-48; G12 = UG1-5-49; G13 = UG1-5-52; G14 = UG1-7-24; G15 = UG2; G16 = UG2-11-03; G17 = UG2-9-01. E1 = Calabar 2016; E2 = Calabar 2017; E3 = Ikom 2016; E4 = Ikom 2017; E5 = Ogoja 2016; E6 = Ogoja 2017

Figure 10 displays the "what-won-where" biplot for rhizome length. The biplot showed that G17 was the winner in Ikom in 2016 (E3), Ikom in 2017 (E4), and Ogoja in 2017 (E6). G14 (UG1-7-24) was the winner in Ogoja in 2016 (E5) while G9 (UG1-5-35) won in Calabar in 2016 (E2). The performances of most of the genotypes were not so different at Calabar in 2016 (E1). G2 (UG1-1107) and G7 (UG1-5-22) performed poorly across all environments.

\subsection{GGE biplot analysis for rhizome yield (t/ha)}

GGE biplot explained $75.9 \%$ of the total variation in rhizome yield due to genotype and GE interactions. PC1 axis explained 58.6\% while the PC2 axis explained 17.3\%. Figure 11 displays the GGE biplot environment vector view for rhizome yield ( $/$ /ha). The biplot showed that Ikom in 2016 (E3) and Ikom in 2017 (E4) were closely correlated and were similar in their interaction pattern. Ogoja in 2016 (E5) and Ogoja in 2017 (E6) were also correlated and similar. Ikom in 2016 (E3) had the longest vector, thus was the most discriminating environment for yield followed by Ikom in 2017 (E4) while Calabar in 2016 (E1) was the least discriminating environment. 


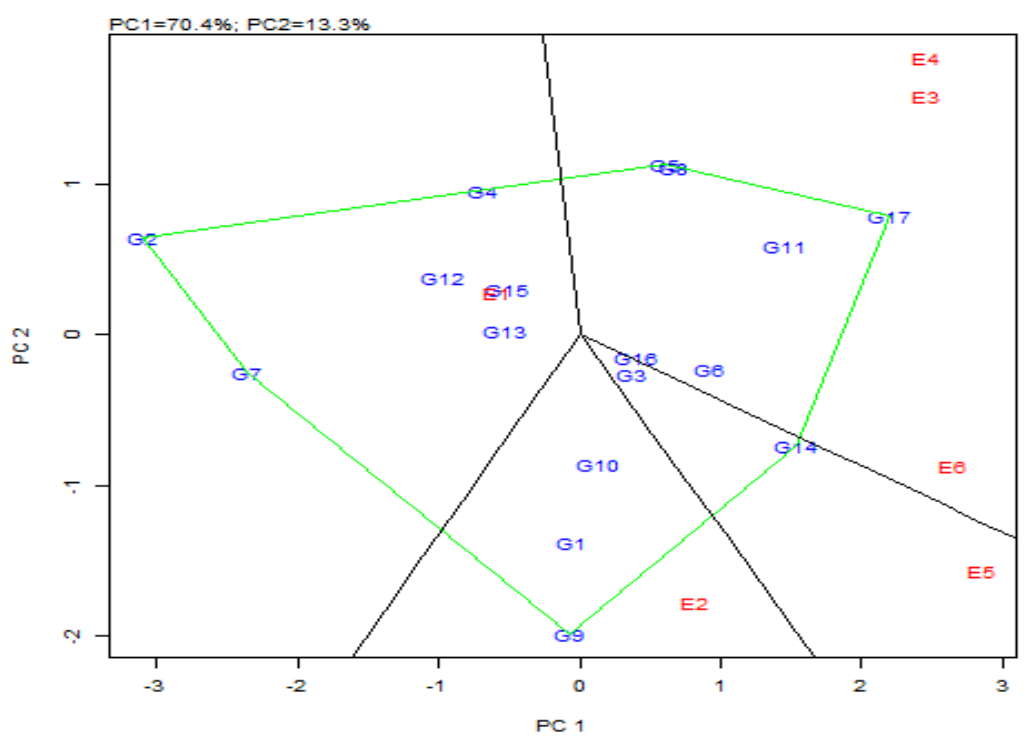

Figure 10: "What-Won-Where" GGE biplot for rhizome length $(\mathrm{cm})$

Key: G1 = UG1; G2 = UG1-11-07; G3 = UG1-13-02; G4 = UG1-2-35; G5 = UG1-5-04; G6 = UG1-5-18; G7 = UG1-5-22; G8 = UG1-5-31; G9 = UG1-5-35; G10 = UG1-5-38; G11 = UG1-5-48; G12 = UG1-5-49; G13 = UG1-5-52; G14 = UG1-7-24; G15 = UG2; G16 = UG2-11-03; G17 = UG2-9-01; E1 = Calabar 2016; E2 = Calabar 2017; E3 = Ikom 2016; E4 = Ikom 2017; E5 = Ogoja 2016; E6 = Ogoja 2017

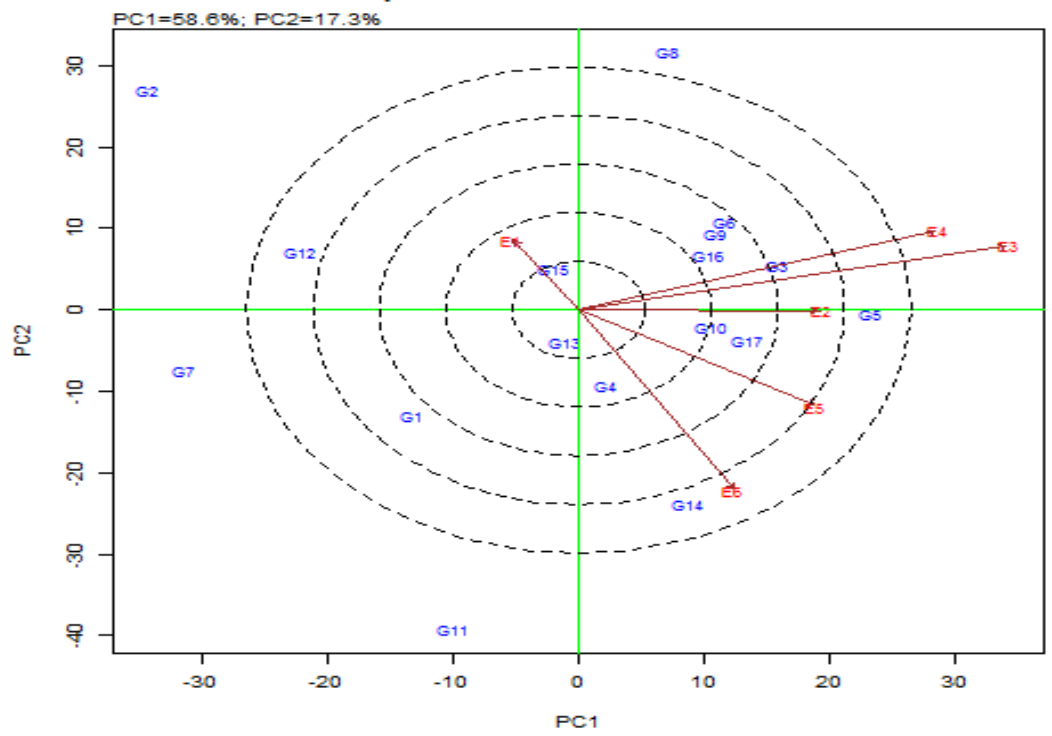

Figure 11: Relationship among the test environments and their discriminating ability for rhizome yield (t/ha)

Key: G1 = UG1; G2 = UG1-11-07; G3 = UG1-13-02; G4 = UG1-2-35; G5 = UG1-5-04; G6 = UG1-5-18; G7 = UG1-5-22; G8 = UG1-5-31; G9 = UG1-5-35; G10 = UG1-5-38; G11 = UG1-5-48; G12 = UG1-5-49; G13 = UG1-5-52; G14 = UG1-7-24; G15 = UG2; G16 = UG2-11-03; G17 = UG2-9-01; E1 = Calabar 2016; E2 = Calabar 2017; E3 = Ikom 2016; E4 = Ikom 2017; E5 = Ogoja 2016; E6 = Ogoja 2017 
Figure 12 displays the GGE biplot environment ranking view for rhizome yield (t/ha). The biplot showed that Calabar in 2016 (E2) was the most representative of the test environments for rhizome yield as its vector had the smallest angle to AEA. However, in the ranking of the test environments, Ikom in 2016 (E3) was ranked closest to the ideal environment while Calabar in 2016 (E1) was ranked least.

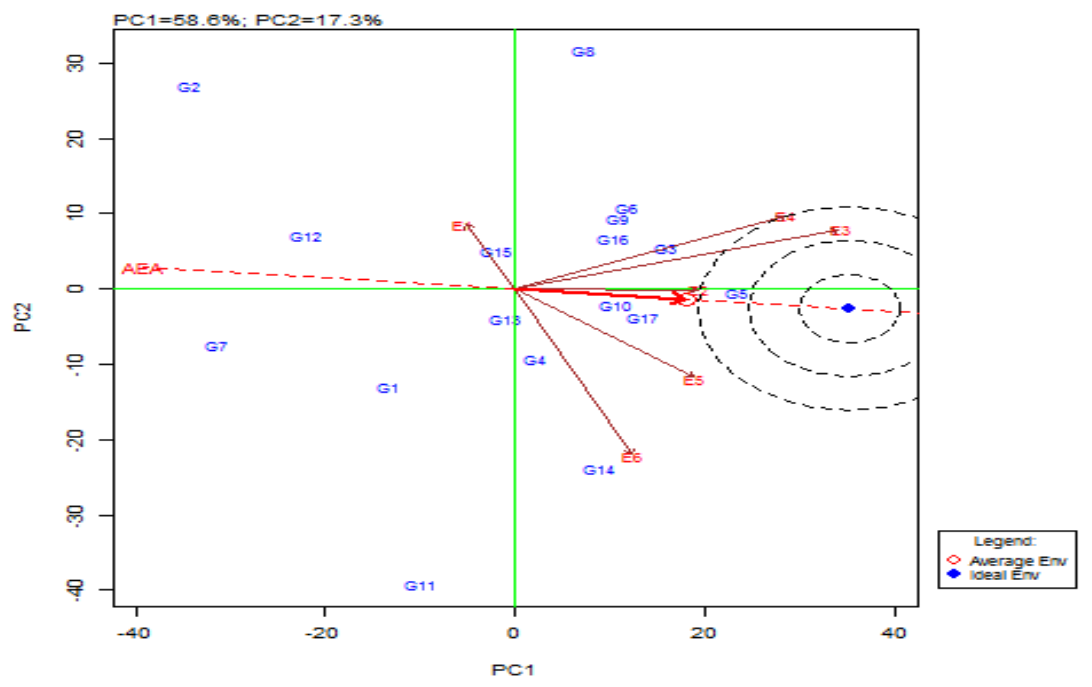

Figure 12: Representativeness and ranking of the environments for rhizome yield ( $t / h a)$

Key: G1 = UG1; G2 = UG1-11-07; G3 = UG1-13-02; G4 = UG1-2-35; G5 = UG1-5-04; G6 = UG1-5-18; G7 = UG1-5-22; G8 = UG1-5-31; G9 = UG1-5-35; G10 = UG1-5-38; G11 = UG1-5-48; G12 = UG1-5-49; G13 = UG1-5-52; G14 = UG1-7-24; G15 = UG2; G16 = UG2-11-03; G17 = UG2-9-01; E1 = Calabar 2016; E2 = Calabar 2017; E3 = Ikom 2016; E4 = Ikom 2017; E5 = Ogoja 2016; E6 = Ogoja 2017

Figure 13 displays the GGE biplot genotype view. The biplot displays the relationship among the genotypes for rhizome yield. The result showed that G5 (UG1-5-04), G16 (UG1-11-03), G17 (UG1-9-01), G10 (UG1-5-38), G6 (UG1-5-18), G3 (UG1-13-02) and G9 (UG1-5-35) had close positive correlations and similar response pattern across the test environments for rhizome yield. G14 (UG1-7-24) and G4 (UG1-2-35) were also positively correlated. G2 (UG1-11-07) had positive correlations with G7 (UG1-5-22), G12 (UG1-5-49), and G1 (UG1) but was negatively correlated with the other genotypes. G11 (UG1-5-48) had negative correlations with G8 (UG1-5-31) while G14 (UG1-7-24) had negative correlations with G15 (UG2) 


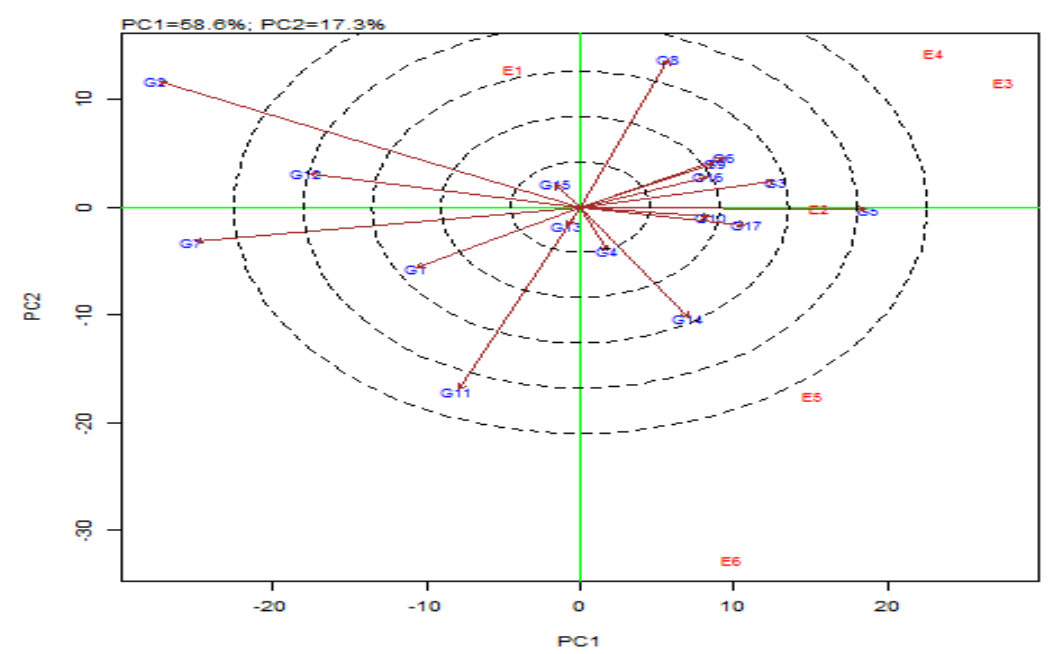

Figure 13: Relationship among the genotype for rhizome yield (t/ha)

Key: G1 = UG1; G2 = UG1-11-07; G3 = UG1-13-02; G4 = UG1-2-35; G5 = UG1-5-04; G6 = UG1-5-18; G7 = UG1-5-22; G8 = UG1-5-31; G9 = UG1-5-35; G10 = UG1-5-38; G11 = UG1-5-48; G12 = UG1-5-49; G13 = UG1-5-52; G14 = UG1-7-24; G15 = UG2; G16 = UG2-11-03; G17 = UG2-9-01; E1 = Calabar 2016; E2 = Calabar 2017; E3 = Ikom 2016; E4 = Ikom 2017; E5 = Ogoja 2016; E6 = Ogoja 2017

The GGE biplot genotype ranking view is displayed in Figure 14. The biplot shows the performance of the genotype with respect to mean yield and stability. G5 (UG1-5-04) had the highest mean yield followed by G17 (UG2-9-01) and both genotypes were relatively stable for yield. G3 (UG1-13-02) and G10 (UG1-5-38) gave the third and fourth best mean yield respectively. G2 (UG1-11-07) and G7 (UG1-5-22) had the poorest mean yield and also exhibited high interactions. In the ranking of the genotypes, G5 (UG1-5-04) was ranked nearest to the "ideal genotype" followed by G17 (UG2-9-01), G3 (UG1-13-02) and G10 (UG1-5-38). Both G2 (UG111-07) and G7 (UG1-5-22) were ranked least.

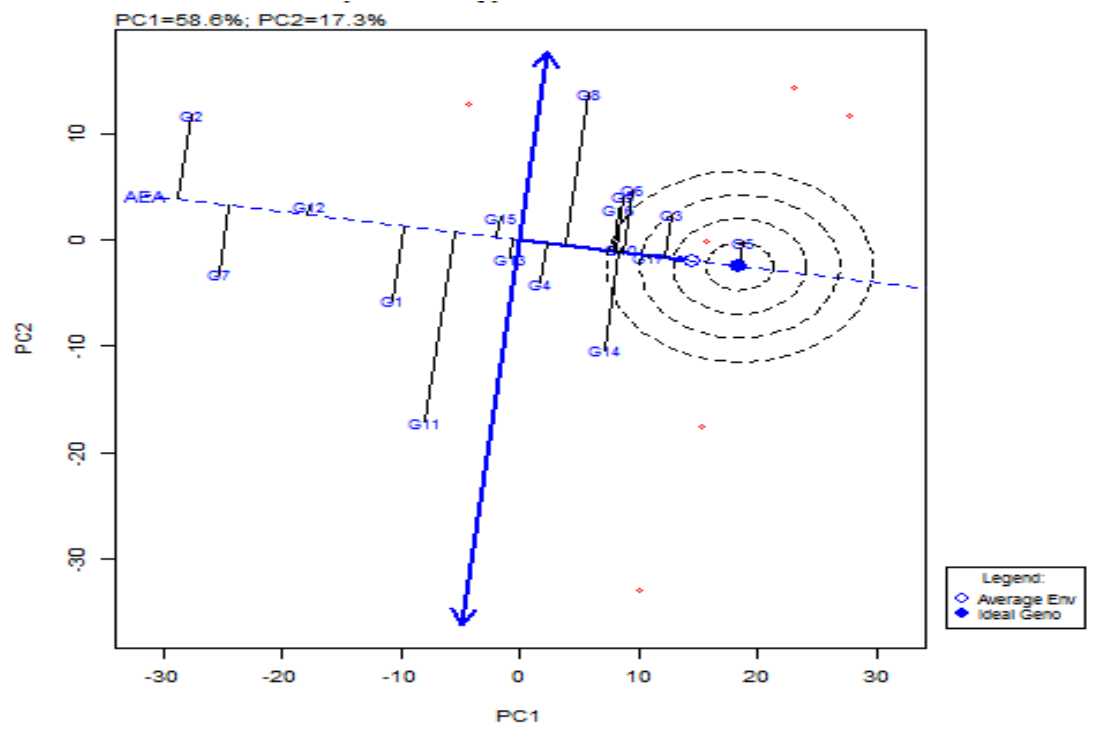

Figure 14: Mean performance and stability for rhizome yield (t/ha) 
Key: G1 = UG1; G2 = UG1-11-07; G3 = UG1-13-02; G4 = UG1-2-35; G5 = UG1-5-04; G6 = UG1-5-18; G7 = UG1-5-22; G8 = UG1-5-31; G9 = UG1-5-35; G10 = UG1-5-38; G11 = UG1-5-48; G12 = UG1-5-49; G13 = UG1-5-52; G14 = UG1-7-24; G15 = UG2; G16 = UG2-11-03; G17 = UG2-9-01. E1 = Calabar 2016; E2 = Calabar 2017; E3 = Ikom 2016; E4 = Ikom 2017; E5 = Ogoja 2016; E6 = Ogoja 2017

Figure 15 displays the "what-won-where" GGE biplot for rhizome yield. The biplot selected G5 (UG1-5-04) as the winner in Calabar in 2016 (E2), Ikom in 2016 (E3).

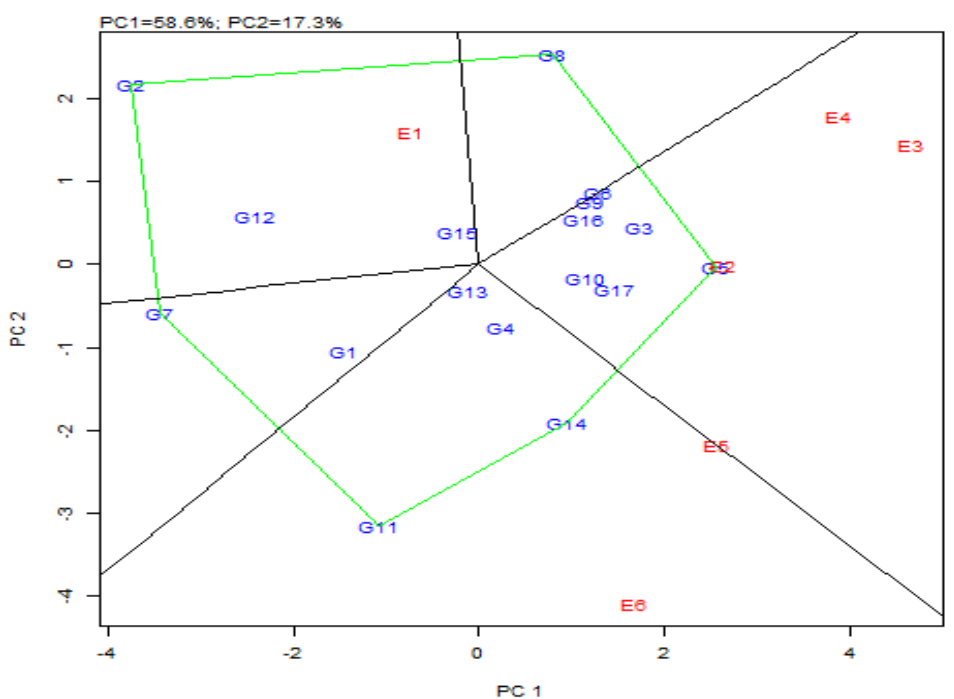

Figure 15: "What-Won-Where" GGE biplot for rhizome yield (t/ha)

Key: G1 = UG1; G2 = UG1-11-07; G3 = UG1-13-02; G4 = UG1-2-35; G5 = UG1-5-04; G6 = UG1-5-18; G7 = UG1-5-22; G8 = UG1-5-31; G9 = UG1-5-35; G10 = UG1-5-38; G11 = UG1-5-48; G12 = UG1-5-49; G13 = UG1-5-52; G14 = UG1-7-24; G15 = UG2; G16 = UG2-11-03; G17 = UG2-9-01; E1 = Calabar 2016; E2 = Calabar 2017; E3 = Ikom 2016; E4 = Ikom 2017; E5 = Ogoja 2016; E6 = Ogoja 2017

Ikom in 2017 (E4) and also shared Ogoja in 2016 (E5) with G14 (UG1-7-24). G11 (UG1-5-48) and 14 (UG1-7-24) were the winners at Ogoja in 2017 (E6). G2 (UG1-11-07) and G7 (UG1-5-22) performed poorly across the six environments. The biplot also identified Ikom (E3 and E4) as the mega environment for G5 (UG1-5-04), G17 (UG2-9-01), G16 (UG2-11-03), G10 (UG1-5-38), G9 (UG1-35), G6 (UG1-5-18) and G3 (UG1-13-02) while Ogoja (E5 and E6) was identified as the mega environment for G11 (UG1-5-48), G14 (UG1-7-24), G13 (UG1-5-52) and G4 (UG1-2-35).

\section{DISCUSSION}

Genotype and genotype by environment (GGE) interaction biplot analysis is an important technique for the selection of genotypes best suited for different environmental conditions; especially since yield trial, data can be daunting. This tool was employed for the evaluation of ginger lines in Calabar, Ikom, and Ogoja in Cross River State, Nigeria. A significant genotype by environment interaction was observed for all the traits studied with cross over interactions observed for some of them. The high significant effect of genotype by environment interaction observed for these traits demonstrates that genotype and environment interaction across the environments plays a meaningful role in breeding adaptable genotypes for wide environments. The variations observed in yield and yield traits of the ginger lines across the locations and years may be attributed to the 
inherent transferable parental trait differences in the ginger lines as well as the environmental influence. Das et al. (2000) also observed significant genotype by environment interactions in some growth and yield traits of ginger. Alghamdi (2004) reported that significant genotype by environment (GE) interactions suggests that across environments, some genotypes were more stable than others. According to Ghaffari and Depao (2005), yield differences attributed to the environment are relevant to genotype evaluation and mega- environment investigations while Samonte et al. (2005) suggested the use of either AMMI or GGE biplots as appropriate tools for analyzing the multiple environment trials data.

According to Yan (2001), the two sources of variation that are relevant to genotype evaluation and should be considered simultaneously for appropriate genotype evaluation are Genotype main effect (primary effects) and genotype by environment interaction (secondary effects). Genotype by environment interaction (GEI) evaluation and stability analysis is an important and efficient tool for plant breeders and agronomists. It helps to identify and select the most stable and high performing genotypes that are suitable under a given set of environmental conditions. According to Edugbo et al. (2015), the evaluation of genotypes in different environments and across years is an essential step in determining their desirability and identifying genotypes with the best mean response across the environments that have a wide scope of adaptation. GGE biplot model was used to evaluate the GEI of the ginger lines as well as their stability across the three locations in the two years (i.e. across six environments). The ginger lines used in this experiment exhibited different levels of stability for the characters evaluated. Their mean performances for the characters varied substantially across the six environments.

\subsection{Discriminating ability and representativeness of test environments}

The purpose of the test-environment evaluation is to identify test environments that effectively identify superior genotypes for a mega-environment. In this study, the GGE biplot model estimated Ikom in 2016 (E3) as the best environment for mean yield and also the most discriminating environment. Test environments that are both discriminating and representative are good test environments for selecting generally adapted genotypes as reported by Yan and Tinker (2006). On the other hand, discriminating but nonrepresentative test environments are useful for selecting specifically adapted genotypes and for culling unstable genotypes. A test location that lacks discriminating ability provides no information about the cultivars (Dehghani et al., 2006; Yan and Tinker, 2006). If a test location is not representative of the target environment, it is not only useless but also misleading since it may provide biased information about the tested genotypes (Dehghani et al., 2006). Therefore, an ideal environment or test location must have discriminating ability (provide much information about the differences among genotypes) and representative (consistent). A representative environment is the most consistent when compared to other test environments and it gives an idea about the stability of the environment. Yan (2002) also stated that the selected environment should have a high ability to discriminate genotypes, as well as the representativeness of the standard growing conditions. Non-discriminating environments provide little information about the genotypes and therefore should not be used as test environments. The existence of close associations among test environments suggests that the same information about the genotypes could be obtained from them; as some of them can be dropped without loss of much information about the genotypes and hence lead to a reduction of the test cost (Yan and Tinker, 2006)

\subsection{Ranking of genotypes based on mean performance and stability}

An ideal genotype should have the highest mean performance and be stable (Karimizadehi et al., 2013). Although such an ideal genotype may not exist in reality, it can be used as a reference for genotype evaluation (Farshadfar et al., 2012). A genotype is more favourable if it is closer to the ideal genotype and the genotypes further away from the ideal genotype are considered unfavourable, therefore, the genotypes with low mean performance and further away from the ideal genotypes are unfavourable. In this study, G2 (UG1-11-07) and G7 (UG1-5-22) were considered unfavourable genotypes in terms of mean performance. Yan and Kang (2003) classified genotypes 
into three categories based on their yield and stability performance: (1) generally adapted: genotypes with high yield and high stability performance (2) specifically adapted: genotypes with high mean yield but low stability performance and (3) adapted nowhere: genotypes with low yield and low stability performance. This classification is similar to the previous work of family behaviour plots by Finlay and Wilkinson (1963) using joint regression. Finlay and Wilkinson classified genotypes into four groups based on their mean performance and stability as follows: Group one is highly desirable (high yielding and high stability) - these genotypes, ranked in descending order), are G17 (UG2-9-01), G11 (UG1-5-48), G5 (UG1-5-04), G3 (UG1-13-02) and G10 (UG1-5-38). The second group which is high yielding but low stability is desirable for specific selection, the genotypes that fall into this group from this study are; G14 (UG1-7-24) and G11 (UG1-5-48), whereas low yield and low stability are possible for special breeding purposes e.g. drought resistance. The genotypes that had both low yield and low stability in this study are G12 (UG1-5-49), G1(UG1), G13(UG1-5-52). The most undesirable group is low yield but high stability, (G2) UG1-11-07, and G7 (UG1-5-22) falls into this group.

\section{3. "Which won where" and mega- environment groupings}

The which won where polygon view shows which genotypes win in which environment. It addresses mega-environment groupings, specific adaptation, and crossover interactions. Yan et al. (2007) reported that responsive genotypes were those having either best or the poorest performance in one or all environments. For rhizome fingers per plant, G17 (UG2-9-01) won in four of the six environments while G14 (UG1-7-24) was the winner in Ogoja in 2016 (E5) and Ogoja in 2017 (E6). G2 (UG1-11-03) and G7 (UG1-5-22) were indicated by the biplot as the poorest performers across all six environments. For rhizome length, G17 was the winner in Ikom in 2016 (E3), Ikom in 2017 (E4), and Ogoja in 2017 (E6). G14 (UG1-7-24) was the winner in Ogoja in 2016 (E5) while G9 (UG1-5-35) won in Calabar in 2016 (E2). The performances of most of the genotypes were not much different at Calabar in 2016 (E1). G2 (UG1-11-07) and G7 (UG1-5-22) performed poorly across all environments. For rhizome yield, G5 (UG1-5-04) won in Calabar in 2016 (E2), Ikom in 2016 (E3), Ikom in 2017 (E4), and also shared Ogoja in 2016 (E5) with G14 (UG1-7-24). G11 (UG1-5-48) and G14 (UG1-7-24) were the winners at E6 (Ogoja 2017). G2 (UG1-11-07) and G7 (UG1-5-22) performed poorly across the six environments. The biplot also identified Ikom (E3 and E4) as a mega environment for G5 (UG1-5-04), G17 (UG2-9-01), G16 (UG2-11-03), G10 (UG1-538), G9 (UG1-35), G6 (UG1-5-18) and G3 (UG1-13-02) while Ogoja (E5 and E6) was identified as a mega environment for G11 (UG1-5-48), G14 (UG1-7-24), G13 (UG1-5-52) and G4 (UG1-2-35). A mega-environment is defined as a location or group of locations that consistently share the same best genotypes (Yan and Tinker, 2006). Mega-environment groupings allow a breeder to recommend genotypes which are good for a particular environment (Gasura et al., 2015). Two mega-environments Ikom (E3 and E4) and Ogoja (E5 and E6) were therefore formed from this study.

\section{SUMMARY, CONCLUSION, AND RECOMMENDATION}

Ginger is an aromatic rhizome crop used as spice and medicine worldwide; the production of the crop cannot meet the demand for it. This research was therefore carried out to evaluate the yield stability of 17 ginger lines as well as identify the ideal environment for ginger cultivation among some test sites using GGE biplot model. GGE biplot models selected UG2-9-01 as the genotype of choice with respect to stability and mean performance for the number of rhizome fingers per plant and rhizome length, UG2-9-01 is therefore recommended as suitable breeding material for the improvement of these traits in ginger.

UG1-5-04 was selected as the "ideal genotype" with respect to rhizome yield. UG2-9-01 was the most stable high performing genotype and was ranked next to UG1-5-04. GGE biplot ranked UG113-02 and UG1-5-38 as 3rd and 4th respectively, therefore these genotypes are recommended for broad cultivation as stable and high yielding genotypes across the three locations used in this study. 
Among the three locations, Ikom was selected as the most discriminating test location and ranked closest to the "ideal test environment", this study, therefore, recommends Ikom as a good test environment for the cultivation of generally adapted ginger genotypes. The study further showed that UG1-5-48 and UG1-7-24 interacted positively with Ogoja in both years. This is an indication of the specific adaptation of these genotypes to the soil and agro-climatic conditions of Ogoja and this suggests the location as favourable for their cultivation.

Funding: This study did not receive any specific financial support.

Competing Interests: The authors declared that they have no conflict of interests.

Contributors/Acknowledgement: The authors acknowledge the invaluable contribution of Dr. Charles

Amadi of the Ginger Research Unit of the National Root Crop Research Institute (NRCRI) Umudike, Abia

State, Nigeria, for making available the ginger lines used for this research.

Views and opinions expressed in this study are the views and opinions of the authors, Asian Journal of Agriculture and Rural Development shall not be responsible or answerable for any loss, damage or liability, etc. caused in relation to/arising out of the use of the content.

\section{References}

Abua, M. N., Ittah, M. A., Obok, E. E., Iwo, G. A., Edugbo, R. E., \& Amadi, C. O. (2020). Nutritional qualities of ginger mutant lines grown in the humid tropical agroecology of Nigeria. Asian Journal of Advances in Agricultural Research, 12(2), 35-42. doi.org/10.9734/ajaar/2020/v12i230080.

Adegboye, M. A. (2011). Evaluation of farmer's response to extension services on ginger production in Kagarko Local Government Area of Kaduna State. Scientific Research and Essays, 6(6), 1166-1171.

Alghamdi, S. S. (2004). Yield stability of some soybean genotypes across diverse environments. Pakistan Journal of Biological Sciences, 7(12), 2109-2114. doi.org/10.3923/pjbs.2004.2109.2114.

Ahmadi, J., Vaezi, B., Shaabani, A., Khademi, K., Fabriki-Ourang, S., \& Pour-Aboughadareh, A. (2015). Non-parametric measures for yield stability in grass pea (Lathyrus sativus L.) advanced lines in semi warm regions. Journal of Agricultural Science and Technology, 17, $1825-1838$.

Babu, K. N., Suraby, E. J., Cissin, J. Minoo, D. Pradeepkumar, T., Parthasarathy, V. A., \& Peter, K.V. (2013). Status of transgenics in Indian spices. Journal of Tropical Agriculture, 51(12), 1-14.

Das, T. T., Pradeepkumar, T., Mayadevi, P., Aiepe, K. C., \& Kumaran, K. (2000). Stability analysis of ginger (Zingiber officinale Rosc). Journal of Spices and Aromatic Crops, 9(2), 165-167.

Dehghani, H., Ebadi A, \& Yousef, A. (2006). Biplot analysis of genotype by environment interaction for barley yield in Iran. Agronomy Journal, 98, 388-393. doi.org/10.2134/agronj2004.0310.

Dehghani, H., Sabaghnia, N., \& Moghaddam, M. (2009). Interpretation of genotype-byenvironment interaction for late maize hybrids' grain yield using a biplot method. Turkish Journal of Agriculture and Forestry, 33, 139-148.

Edugbo, R. E., Nwofia, G. E., \& Fayeun, L. S. (2015). An assessment of soybean (Glycine max L. Merrill) yield in different environments using AMMI and GGE biplot models in humid rainforest fringes of Nigeria. Agricultura Tropica et Subtropica, 48(3), 82-90. doi.org/10.1515/ats-2015-0012.

FAO (2006). Food and Agriculture Organization of the United Nations. Ginger: Post-production management for improved market Access. Prepared by Anne Plotto. Edited by François Mazaud, Alexandra Röttger, Katja Steffel. Food and Agriculture Organization of the United Nations (FAO), AGST. 21 pp.

FAO (2017). Food and Agriculture Organization. Production quantity of ginger in metric tonnes. Available: http//www.fao.org (accessed on August 24th 2018 ). 
Farshadfar, E., Mohammadi, M. P., \& Maryam, J. (2012). Evaluation of phenotypic stability in bread wheat genotypes using GGE-biplot. International Journal of Agriculture and Crop Science, 4, 904-910.

Finlay, K. W., \& Wilkinson G. N. (1963). The analysis of adaptation in a plant breeding programme. Australian Journal of Agricultural Research, 14, 742-754.

Gabriel, K. R. (1971). The biplot graphic display of matrices with application to principal component analysis. Biometrika, 58, 453-467. doi.org/10.1093/biomet/58.3.453.

Gasura, E., Setimela, P. S., \& Souta, C. M. (2015). Evaluation of the performance of sorghum genotypes using GGE biplot. Canadian Journal of Plant Science, 95, 1205-1214. doi.org/10.4141/cjps-2015-119.

Ghaffari, A. A., \& Depao, E. (2005). Agro-climatic zoning of Iranian cold drylands. In: Proceedings of the 2006 Iranian Crop Science Congress, pp. 42-48.

Karimizadehi, R, Mohtasham, M. N, Sabaghn, A. A, Mahmood, B., \& Roustami, F. (2013). GGE biplot analysis of yield stability in multi-environment trials of lentil genotypes under rainfed condition. Notulae Scientia Biologicae, 5, 256-262.

Mohamed, N. E. (2013). Genotype by environment interactions for grain yield in bread wheat (Triticum aestivum L.). Journal of Plant Breeding and Crop Science, 7, 150-157.

Odor, E. O., Iwo, G. A., \& Obok, E. E. (2017). Graphical assessment of yield stability and adaptation of cucumber (Cucumis sativus L.) genotypes in Cross River State, Nigeria. Journal of Agricultural and Crop Research, 5(6), 108-116.

Oliveira, T. R. A., Carvalho, H. W. L., Costa, E. F. N., \& Carvalho, F. J. L.S. (2017). Correlation among adaptability and stability assessment models in maize cultivars. Australian Journal of Crop Science, 11, 516-521. doi.org/10.21475/ajcs.17.11.05.p304.

Oyekunle, M., Haruna, A., Badu-Apraku, B., Usman, I. S., Mani, H., Ado, S. G., Olaoye, G., Obeng-Antwi, K., Abdulmalik, R. O., \& Ahmed, H. O. (2017). Assessment of earlymaturing maize hybrids and testing sites using GGE biplot analysis. Crop Science, 57, 2942-2950. doi.org/10.2135/cropsci2016.12.1014.

Peter, K. V., Ravindran, P. N., Divakaran, M., \& Babu, K.N. (2007). Horticulture: Vegetable, Tuber and Spice Crops. http://nsdl.niscair.res.in/jspui/bitstream/123456789/471/1/revised\%20Breeding\%20of\%20 spices.pdf.

Samonte, S. O. P. B., Wilson, L. T., McClung, A., \& Medley, J. C. (2005). Targeting cultivars onto rice growing environments using AMMI and SREG GGE biplot analysis. Crop Science 45, 2414-2424.

Santos, A. D., Amaral-Júnior, A. T. D., Kurosawa, R. D. N. F., Gerhardt, I. F. S., \& Fritsche, Neto, R. (2017). GGE Biplot projection indiscriminating the efficiency of popcorn lines to use nitrogen. Ciência e Agrotecnologia, 41(1), 22-31. doi.org/10.1590/141370542017411030816

Vaezi, B., Pour-Aboughadareh, A., Mohammadi, R., Armion, M., Mehraban, A., Hossein-Pour, T., \& Dorii, M. (2017). GGE biplot and AMMI analysis of barley yield performance in Iran. Cereal Research Communications, 45(3), 500-511.

Yan, W., Hunt, L. A., Sheng, W. Q., \& Szlavnics, Z. (2000). Cultivar evaluation and mega environment investigation based on the GGE biplot. Crop Science, 40, 597-605

Yan, W., Cornelius, P. L., Crossa, J., \& Hunt, L. A. (2001). Two types of GGE biplots for analyzing multi-environment trial data. Crop Science, 41, 656-663.

Yan, W. K. (2001). GGE biplot: A windows application for graphical analysis of multienvironment trial data and other types of two-way data. Agronomy Journal, 93, 11111118.

Yan, W., \& Kang, M. S. (2003). GGE biplot analysis: a graphical tool for breeders, geneticists and agronomists. Florida, CRC Press, 267pp.

Yan, W., \& Tinker, N. A. (2006). Biplot analysis of multi-environment trial data: Principles and applications. Canadian Journal of Plant Science, 86, 623-645. doi.org/10.1201/9781420040371-5. 
Yan, W. K., Kang, M. S., Ma, B., Woods, S., \& Cornelius, P. L. (2007). GGE Biplot vs. AMMI analysis of genotype-by-environment data. Crop Science, 47, 643-655.

Yan, W. (2014). Crop variety trials: Data management and analysis. Pg 349. John Wiley and Sons, New Jersey, USA.

Zahia, K., Farrah, A., \& Hamenna, B. (2010). Analysis of the genotype x environment interaction of barley grain yield (Hordeum vulgare L.) under semi-arid conditions. Advances in Environmental Biology, 4(1), 34-40. 